\title{
Cyclooxygenase-2 inhibitors modulate skin aging in a catalytic activity-independent manner
}

\author{
Mi Eun Lee', So Ra Kim', Seungkoo Lee ${ }^{2}$, \\ Yu-Jin Jung ${ }^{3}$, Sun Shim $\mathrm{Choi}^{4}$, \\ Woo Jin $\mathrm{Kim}^{5}$ and Jeong A Han ${ }^{1,6}$ \\ ${ }^{1}$ Department of Biochemistry and Molecular Biology \\ Institute of Medical Sciences \\ ${ }^{2}$ Department of Anatomic Pathology \\ Kangwon National University Hospital \\ Kangwon National University School of Medicine \\ ${ }^{3}$ Department of Biological Sciences \\ College of Natural Sciences \\ ${ }^{4}$ Department of Medical Biotechnology \\ College of Biomedical Science \\ Institute of Bioscience and Biotechnology \\ Kangwon National University \\ ${ }^{5}$ Department of Internal Medicine \\ Kangwon National University School of Medicine \\ Chuncheon 200-701, Korea \\ ${ }^{6}$ Corresponding author: Tel, 82-33-250-8832; \\ Fax, 82-33-255-8809; E-mail, gshja@kangwon.ac.kr \\ http://dx.doi.org/10.3858/emm.2012.44.9.061
}

Accepted 29 June 2012

Available Online 6 July 2012

Abbreviations: COX-2, cyclooxygenase-2; NSAID, non-steroidal anti-inflammatory drug

\begin{abstract}
It has been proposed that the pro-inflammatory catalytic activity of cyclooxygenase-2 (COX-2) plays a key role in the aging process. However, it remains unclear whether the COX-2 activity is a causal factor for aging and whether COX-2 inhibitors could prevent aging. We here examined the effect of COX-2 inhibitors on aging in the intrinsic skin aging model of hairless mice. We observed that among two selective COX-2 inhibitors and one non-selective COX inhibitor studied, only NS-398 inhibited skin aging, while celecoxib and aspirin accelerated skin aging. In addition, NS-398 reduced the expression of p53 and p16, whereas celecoxib and aspirin enhanced their expression. We also found that the aging-modulating
\end{abstract}

\begin{abstract}
effect of the inhibitors is closely associated with the expression of type I procollagen and caveolin-1. These results suggest that pro-inflammatory catalytic activity of COX-2 is not a causal factor for aging at least in skin and that COX-2 inhibitors might modulate skin aging by regulating the expression of type I procollagen and caveolin-1.
\end{abstract}

Keywords: caveolin-1; cyclooxygenase inhibitors; procollagen; skin aging

\section{Introduction}

Prostaglandin endoperoxide synthase (PTGS), also called cyclooxygenase (COX), is responsible for the conversion of arachidonic acid to prostaglandin $\mathrm{H}_{2}$ $\left(\mathrm{PGH}_{2}\right)$, which is the common precursor for the biosynthesis of various prostanoids including prostaglandin $\mathrm{D}_{2}\left(\mathrm{PGD}_{2}\right)$, prostaglandin $\mathrm{E}_{2}\left(\mathrm{PGE}_{2}\right)$, prostaglandin $\mathrm{I}_{2}\left(\mathrm{PGI}_{2}\right)$ and thromboxane $\mathrm{A}_{2}\left(\mathrm{TXA}_{2}\right)$. Among them, $\mathrm{PGE}_{2}$ and $\mathrm{PGI}_{2}$ are known to be important inflammatory mediators. There are two isozymes of COX, COX-1 and COX-2. In general, COX-1 is a constitutive type, whereas COX-2 is an inducible type whose expression is increased by various stimuli (Smith et al., 1996; Funk, 2001; Rouzer and Marnett, 2008; Park and Kwon, 2011).

Most non-steroidal anti-inflammatory drugs (NSAIDs) are COX inhibitors, which occupy the active site of COX to inhibit the enzyme's catalytic activity. Traditionally used NSAIDs including aspirin, ibuprofen and indomethacin inhibit the catalytic activity of both COX-1 and COX-2 non-selectively. In contrast, recently developed NSAIDs such as NS-398, celecoxib or rofecoxib inhibit the catalytic activity of COX-2 selectively (Flower, 2003).

While underlying mechanisms of aging have not been fully elucidated, it has been proposed that the pro-inflammatory catalytic activity of COX-2 plays a crucial role in the aging process. Reactive oxygen species (ROS) generated in the process of normal metabolism or inflammation have been suggested to activate the redox-sensitive transcription factor $\mathrm{NF}-\kappa \mathrm{B}$ inducing the expression of pro-inflammatory genes such as COX-2, which in turn stabilizes 
chronic inflammatory status by producing ROS to cause tissue damage and aging (Chung et al., 2009).

If the pro-inflammatory catalytic activity of COX-2 plays a causal role for aging, COX-2 inhibitors will have to inhibit the aging process. However, it has not yet been determined whether the catalytic activity of COX-2 is involved in the aging process and whether COX-2 inhibitors prevent aging. Actually, expression levels of COX-2 are observed to increase in heart and kidney of aged rats (Kim et al., 2000; Kim et al., 2001), but not in brain of aged rats and endothelial cells of aged humans (Baek et al., 2001; Sanguino et al., 2004; Donato et al., 2008). In addition, non-selective COX inhibitors such as acetylsalicylic acid, salicylic acid and indomethacin do not affect or rather shorten the life span in drosophila (Massie et al., 1985). A recent report shows that celecoxib, a selective COX-2 inhibitor, extends the life span in $C$. elegans, which is mediated by a COX-2 catalytic activity-independent mechanism (Ching et al., 2011).

In the present study, to elucidate whether the catalytic activity of COX-2 is a causal factor for aging, we analyzed the effect of COX-2 inhibitors on aging in the intrinsic skin aging model of hairless mice. Our data shows that NS-398 (a selective COX-2 inhibitor) inhibits skin aging, while celecoxib (a selective COX-2 inhibitor) and aspirin (a non-selective COX inhibitor) accelerate skin aging. In addition, the aging-modulating effect of the inhibitors was associated with the expression of p53, p16, type I procollagen and caveolin-1.

\section{Results}

\section{COX-2 inhibitors modulate intrinsic skin aging in a catalytic activity-independent manner}

To elucidate whether the COX-2 catalytic activity is a causal factor for intrinsic skin aging, we analyzed the effect of two selective COX-2 inhibitors (NS-398 and celecoxib) and one non-selective COX inhibitor (aspirin) on intrinsic skin aging in SKH-1 hairless mice. The $\mathrm{IC}_{50}$ values of NS-398, celecoxib and aspirin had been reported for recombinant human or ovine COX-1 and COX-2 (Barnett et al., 1994; Johnson et al., 1995). In addition, we had previously established that around 10 -fold higher concentrations of $\mathrm{IC}_{50}$ of NS-398 and celecoxib $(20 \mu \mathrm{M}$ and $0.5 \mu \mathrm{M}$, respectively) as well as $\mathrm{IC}_{50}$ of aspirin $(1.0 \mathrm{mM})$ inhibited the COX-2 catalytic activity significantly without cellular toxicity in human dermal fibroblasts (HDFs). We had used $\mathrm{IC}_{50}$ for aspirin because 10 -fold higher concentration caused acute cellular toxicity (Kim et al., 2008). In the present study, we used 5-fold and 50-fold higher concentrations for each drug than the concentrations used in HDFs to inhibit the COX-2 catalytic activity sufficiently in in vivo skin (Table 1).

Intrinsic skin aging is characterized by thinning, sagging and wrinkling of the skin (Rittie and Fisher, 2002). Thus, we applied the inhibitors everyday onto the right and left side of dorsal skin of the mice for 12 weeks and measured their skin fold thickness by using a caliper. The data showed that skin fold thickness was decreased by $\sim 20 \%$ after 12 weeks when compared to 0 weeks under the treatment of the vehicle (ethanol: propylene glycol = 7:3), which was significantly prevented by the treatment of NS-398 (Figure 1A). In contrast, celecoxib and aspirin further decreased the skin fold thickness as compared to the vehicle (Figures 1B and 1C).

Skin is composed of two layers, the epidermis and the dermis, whose thickness has been known to decrease in the intrinsic skin aging (Varani et al., 2000). Therefore, we obtained skin tissues at the end of the drug treatment for 12 weeks to measure epidermal thickness. It was observed that the treatment of NS-398 increased epidermal thickness (Figures 2A and 2D), whereas the treatment of celecoxib and aspirin decreased epidermal thickness as compared to the treatment of the vehicle (Figures 2B-2D). These results show that NS-398 inhibits the aging-associated thinning of the skin while celecoxib and aspirin accelerate it.

We then analyzed the effect of the inhibitors on wrinkling of the skin by using skin replica. The data showed that the treatment of NS-398 greatly reduced average wrinkle depth as compared to the treatment of the vehicle (Figures 3A and 3D). On the contrary, celecoxib treatment significantly increased average wrinkle depth as compared to the treatment of the vehicle (Figures 3B and 3D). In the case of aspirin, we observed that the average wrinkle depth was prominently increased by the treatment of 50 $\mathrm{mM}$ aspirin but not by the treatment of $5 \mathrm{mM}$ aspirin (Figures $3 \mathrm{C}$ and $3 \mathrm{D}$ ). The maximum wrinkle depth and average wrinkle area were also measured in the same replica and the data showed exactly the same tendency with the average wrinkle depth (data not shown). These results indicate that NS-398 inhibits

Table 1. $I C_{50}$ values of COX-2inhibitors and used concentrations

\begin{tabular}{lcccc}
\hline Inhibitors & $\begin{array}{c}\mathrm{IC}_{50} \text { for } \\
\text { COX-1 }\end{array}$ & $\begin{array}{c}\mathrm{IC}_{50} \text { for } \\
\text { COX-2 }\end{array}$ & $\begin{array}{c}\text { Used doses } \\
\text { in HDFs }\end{array}$ & $\begin{array}{c}\text { Used doses } \\
\text { in mice }\end{array}$ \\
\hline NS-398 & $75 \mu \mathrm{M}$ & $1.77 \mu \mathrm{M}$ & $20 \mu \mathrm{M}$ & $0.1 / 1.0 \mathrm{mM}$ \\
Celecoxib & $15 \mu \mathrm{M}$ & $0.04 \mu \mathrm{M}$ & $0.5 \mu \mathrm{M}$ & $2.5 / 25 \mu \mathrm{M}$ \\
Aspirin & $0.75 \mathrm{mM}$ & $1.25 \mathrm{mM}$ & $1.0 \mathrm{mM}$ & $5 / 50 \mathrm{mM}$ \\
\hline
\end{tabular}


A

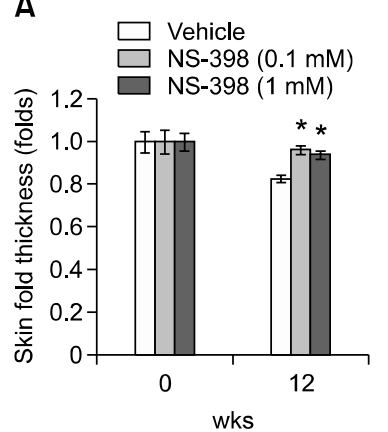

C

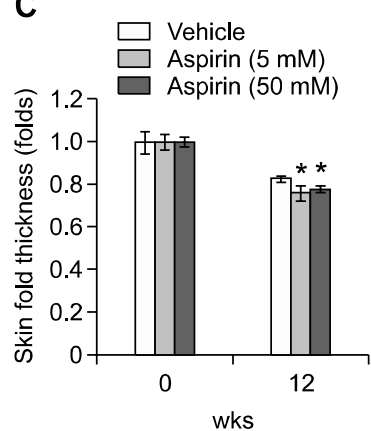

Figure 1. NS-398 increases but celecoxib and aspirin decrease skin fold thickness in hairless mice. NS-398 (A), celecoxib (B) and aspirin (C) were treated to the right and left side of dorsal skin of mice for 12 weeks. Skin fold thickness was measured by using a caliper. Data represent means \pm S.E. $(n=5)$. ${ }^{*} P<0.05$; compared with the corresponding vehicle-treated group.

the aging-associated wrinkling of the skin, whereas celecoxib and high dose of aspirin accelerate it.

Collectively, these results demonstrate that NS-398 inhibits the intrinsic skin aging while celecoxib and aspirin accelerate it, suggesting that the catalytic activity of COX-2 does not mediate intrinsic skin aging and that COX-2 inhibitors modulate intrinsic skin aging through a catalytic activity-independent mechanism.

\section{The aging-modulating effect of COX-2 inhibitors is associated with p53 and p16 expression}

It is widely accepted that diverse stimuli inducing cellular senescence ultimately activate either or both of p53 and p16/pRB pathway. Although it has not been well established that these pathways are also critical channels for individual aging, accumulating evidence indicates that the p53 and p16/pRB pathways are common pathways in individual aging, too (Campisi, 2005).

Therefore, to support the aging-modulating effect of the inhibitors on the skin aging at molecular levels, we examined expression levels of p53 and p16 under the treatment of the inhibitors. The
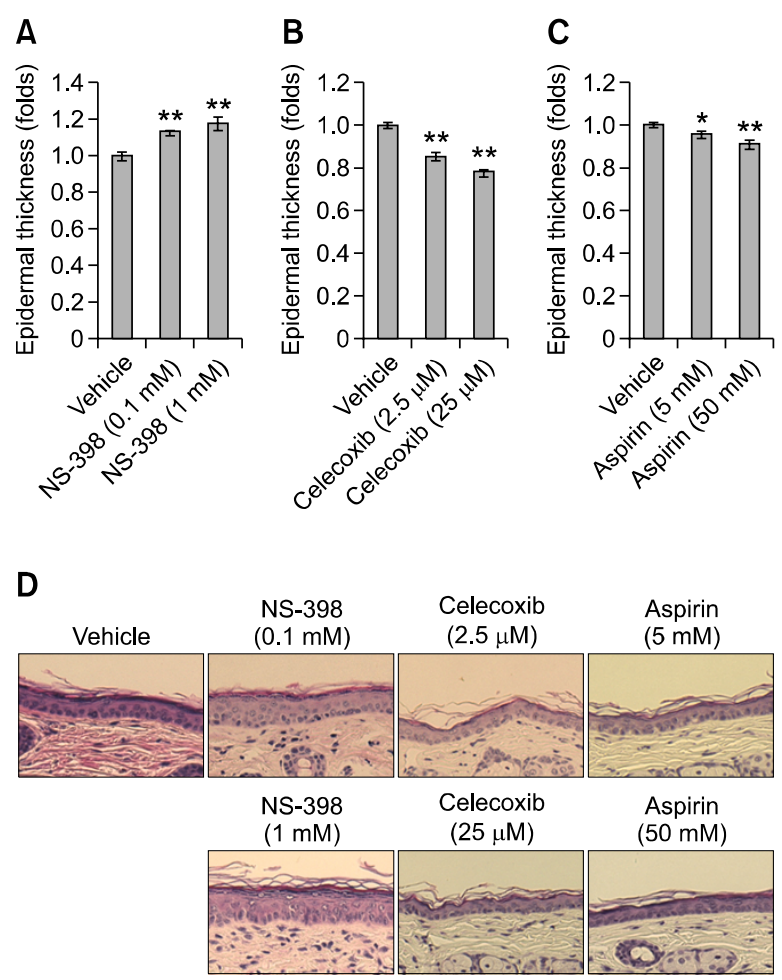

Figure 2. NS-398 increases but celecoxib and aspirin decrease epidermal thickness in hairless mice. NS-398 (A), celecoxib (B) and aspirin (C) were treated to the right and left side of dorsal skin of mice for 12 weeks. Paraffin sections of the skin were stained with $\mathrm{H} \& \mathrm{E}$ and then epidermal thickness was measured. Data represent means \pm S.E. $(n=$ 10). ${ }^{*} P<0.05$ and ${ }^{* *} P<0.01$; compared with the corresponding vehicle-treated group. (D) Representative images of H \& E staining were shown.

western blotting data showed that NS-398 inhibited expression of both p53 and p16 dose-dependently (Figures $4 \mathrm{~A}$ and $4 \mathrm{D}$ ), while celecoxib and aspirin enhanced the expression of p53 and p16 dose-dependently as compared to the vehicle (Figures 4B-4D). These results suggest that COX-2 inhibitors modulate the skin aging through both $\mathrm{p} 53$ and $\mathrm{p} 16 / \mathrm{pRB}$ pathways, and support our conclusion at molecular levels that NS-398 inhibits intrinsic skin aging whereas celecoxib and aspirin accelerate it.

\section{The aging-modulating effect of COX-2 inhibitors is associated with type I procollagen expression}

Skin is predominantly composed of type I collagen, which is synthesized in dermal fibroblasts and secreted as the procollagen form. The expression of type I procollagen is greatly reduced in intrinsic skin aging, which is thought to be a crucial factor for degenerative changes such as thinning and wrinkling of the skin (Chung et al., 2001; Rittié and Fisher, 2002; Shoulders and Raines, 2009).

To test the possibility that COX-2 inhibitors might 
A

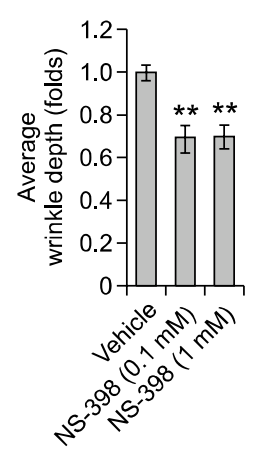

B

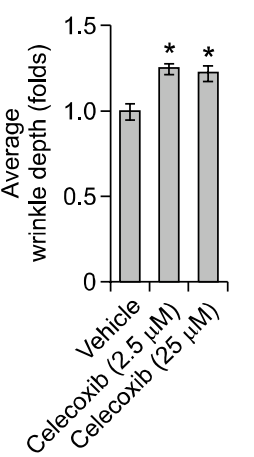

C

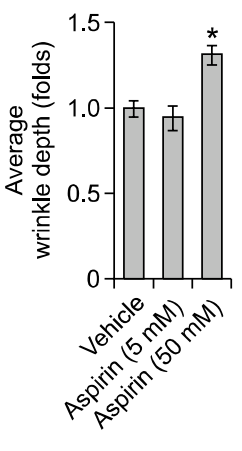

D
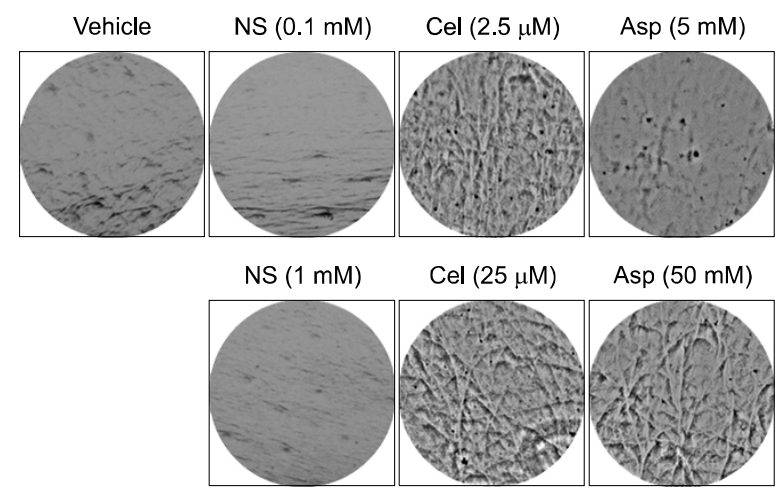

Figure 3. NS-398 decreases but celecoxib and high dose of aspirin increase average wrinkle depth in hairless mice. NS-398 (A), celecoxib (B) and aspirin (C) were treated to the right and left side of dorsal skin of mice for 12 weeks. Skin replicas were obtained and average wrinkle depth was measured. Data represent means \pm S.E. $(n=10) .{ }^{*} P<$ 0.05 and ${ }^{* *} P<0.01$; compared with the corresponding vehicle-treated group. (D) Representative images of skin replicas were shown.

regulate the expression of type I procollagen in the skin, we monitored protein levels of type I procollagen under the treatment of the inhibitors. The western blotting data showed that NS-398 markedly increased the amount of type I procollagen dose-dependently (Figures 5A and 5D), whereas celecoxib and aspirin decreased the amount of type I procollagen dosedependently as compared to the vehicle (Figures $5 B-5 D)$. These results demonstrate that the agingmodulating effect of the inhibitors is associated with type I procollagen expression, suggesting that COX-2 inhibitors might modulate intrinsic skin aging via regulation of type I procollagen expression.

\section{The aging-modulating effect of COX-2 inhibitors is associated with caveolin-1 expression}

Caveolin-1, a structural component of caveolae in the plasma membrane, has been proposed to be an important mediator for cellular senescence (Cho

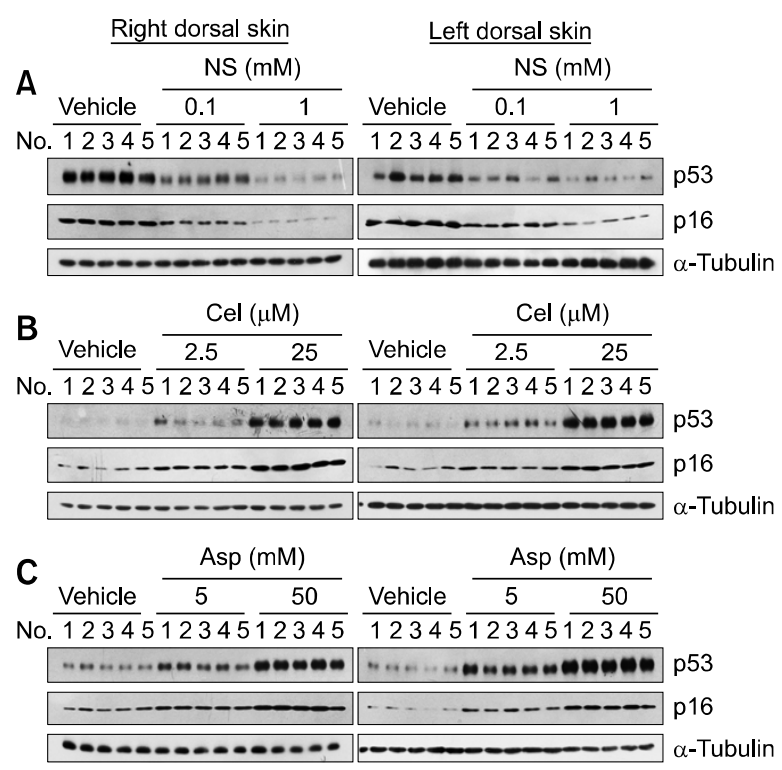

D
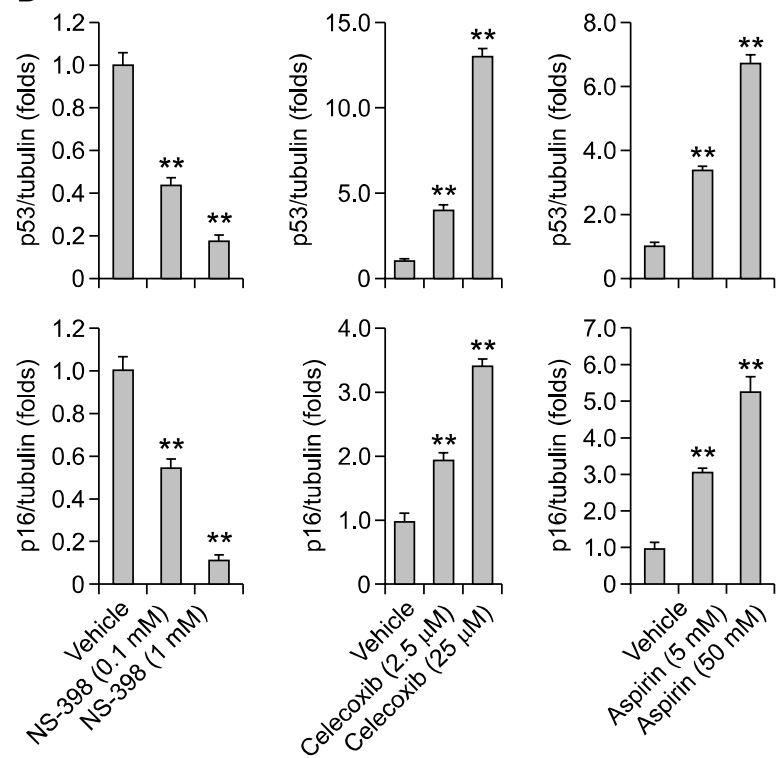

Figure 4. NS-398 decreases but celecoxib and aspirin increase the expression of p53 and p16. NS-398 (A), celecoxib (B) and aspirin (C) were treated to the right and left side of dorsal skin of mice for 12 weeks. Skin lysates were prepared from the indicated numbered mice and western blot analysis was carried out using antibodies against p53 and p16. $\alpha$-Tubulin was used as a loading control. Exposing times are variable to acquire the best quality of the data. (D) Quantitative analysis for p53 and p16 expression was done by densitometry. Data represent means \pm S.E. $(n=10) .{ }^{* *} P<0.01$; compared with the corresponding vehicle-treated group.

and Park, 2005). In addition, we had previously observed that the treatment of COX-2 inhibitors altered the expression of caveolin-1 in HDFs (Kim et al., 2008).

Therefore, to test the possibility that the inhibitors might regulate caveolin-1 expression in the skin 

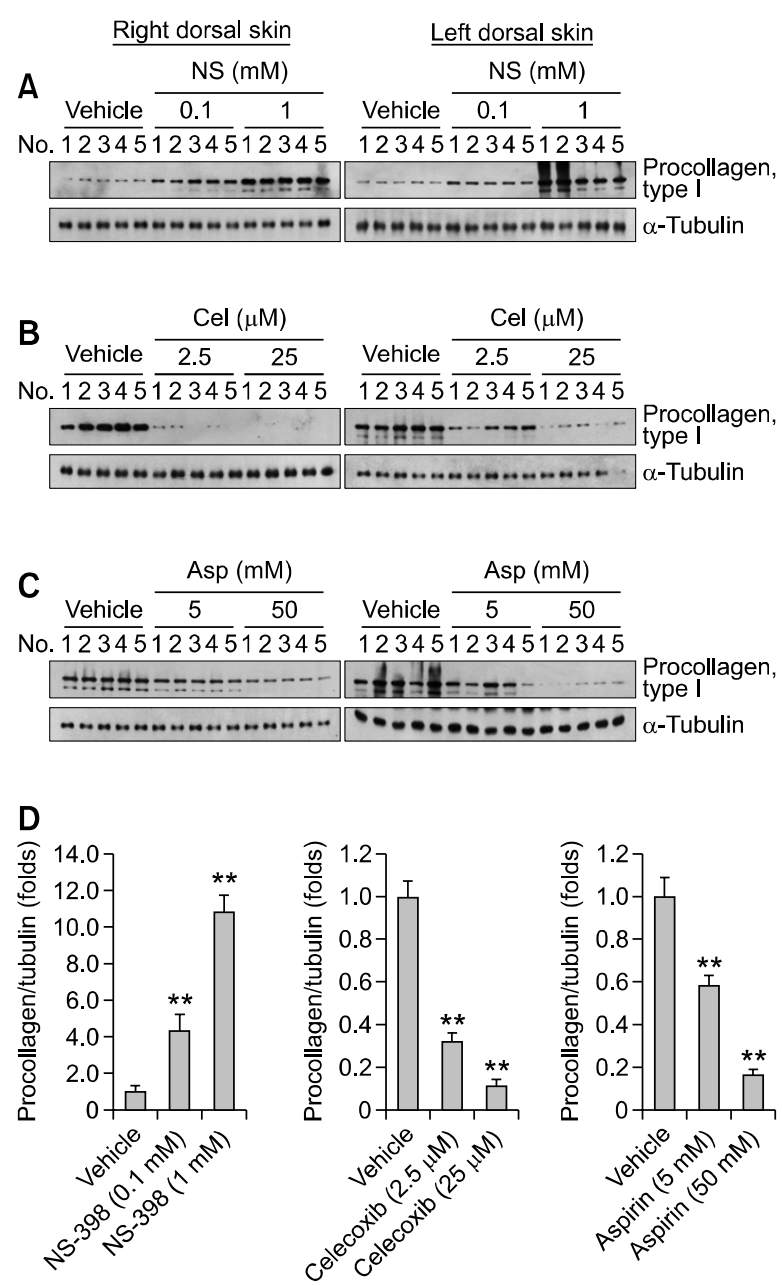

Figure 5. NS-398 increases but celecoxib and aspirin decrease the expression of type I procollagen. NS-398 (A), celecoxib (B) and aspirin (C) were treated to the right and left side of dorsal skin of mice for 12 weeks. Skin lysates were prepared from the indicated numbered mice and western blot analysis was carried out using an antibody for type I procollagen. $\alpha$-Tubulin was used as a loading control. Exposing times are variable to acquire the best quality of the data. (D) Quantitative analysis for type I procollagen expression was done by densitometry. Data represent means \pm S.E. $(n=10) .{ }^{* *} P<0.01$; compared with the corresponding vehicle-treated group.

aging, we monitored expression levels of caveolin-1 under the treatment of the inhibitors. As shown in Figures 6A and 6D, NS-398 decreased the amount of caveolin-1 dose-dependently as compared to the vehicle. On the contrary, celecoxib and aspirin increased the amount of caveolin-1 dose-dependently as compared to the vehicle (Figures 6B-6D). These results demonstrate that the aging-modulating effect of the inhibitors is associated with caveolin-1 expression, suggesting that COX-2 inhibitors might modulate intrinsic skin aging via regulation of caveolin-1 expression.
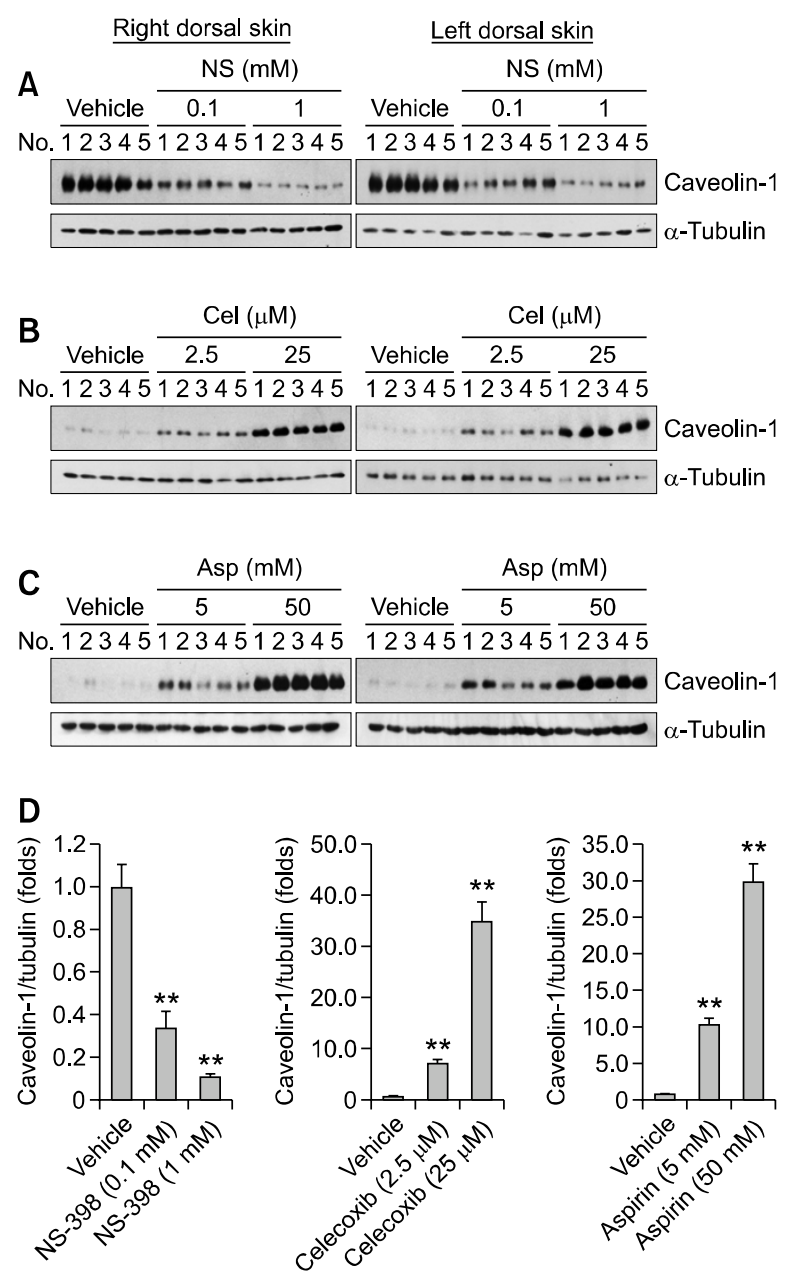

Figure 6. NS-398 decreases but celecoxib and aspirin increase the expression of caveolin-1. NS-398 (A), celecoxib (B) and aspirin (C) were treated to the right and left side of dorsal skin of mice for 12 weeks. Skin lysates were prepared from the indicated numbered mice and western blot analysis was carried out using an anti-caveolin- 1 antibody. $\alpha$-Tubulin was used as a loading control. Exposing times are variable to acquire the best quality of the data. (D) Quantitative analysis for caveolin-1 expression was done by densitometry. Data represent means \pm S.E. $(n=$ 10). ${ }^{* \star} P<0.01$; compared with the corresponding vehicle-treated group.

\section{Discussion}

The pro-inflammatory catalytic activity of cyclooxygenase-2 (COX-2) has been proposed to be a crucial factor for individual aging as well as cellular senescence (Zdanov et al., 2007; Chung et al., 2009). In the present study, we have investigated whether COX-2 inhibitors could prevent aging in the intrinsic skin aging model of hairless mice. Our data showed that among three COX-2 inhibitors studied, only NS-398 inhibits the skin aging while celecoxib and aspirin accelerate it (Figures 1-3), suggesting that the catalytic activity of COX-2 does not mediate aging at least in skin. Consistent with these results, 
we had previously observed that among six COX-2 inhibitors studied, only NS-398 inhibited cellular senescence whereas two selective COX-2 inhibitors (celecoxib and nimesulide) and three non-selective COX inhibitors (aspirin, ibuprofen and flurbiprofen) accelerated the senescence in human dermal fibroblasts (HDFs) (Kim et al., 2008).

It is still controversial whether cellular senescence is a source for individual aging. At least in skin, however, senescent fibroblasts have been detected in the dermis and their numbers have been found to increase with age in mice, baboons and humans (Dimri et al., 1995; Herbig et al., 2006; Wang et al., 2009). According to our present and previous studies, the aging-modulating effect of COX-2 inhibitors in in vivo skin is consistent with the senescence-modulating effect of the inhibitors in dermal fibroblasts (Figures 1-3; Kim et al., 2008). These findings support the idea that cellular senescence is a source for individual aging, and suggest that modulation of cellular senescence might be a good strategy to modulate individual aging.

Cellular senescence can be induced by diverse stimuli including dysfunctional telomeres, DNA damage, oncogenes and others, which ultimately activate either or both of the p53 and p16/pRb pathways. In general, the activity of p53 increases in the course of cellular senescence with or without an elevation of p53 expression (Atadja et al., 1995; Kulju and Lehman, 1995). The p16 expression is usually enhanced in cellular senescence (Alcorta et al., 1996). Compatible with these results, expression levels of p53 increases in brain of aged rats (Dorszewska and Adamczewska-Goncerzewicz, 2004). In the case of heart, kidney, liver and lung, the total protein amount of p53 is not changed but the amount of acetylated p53 increases in aged rats (Braidy et al., 2011). On the other hand, the p16 expression increases in most tissues of aged mice and rats (Krishnamurthy et al., 2004). These studies suggest that the p53 and p16/pRb pathways are ultimate channels for individual aging, too. We observed here that the expression of p53 and p16 was changed by the treatment of COX-2 inhibitors, which was in agreement with the aging-modulating effect of the inhibitors (Figure 4). These data suggest that COX-2 inhibitors modulate skin aging by affecting both p53 and p16/pRb pathways and strongly support our conclusion at molecular levels that NS-398 inhibits intrinsic skin aging whereas celecoxib and aspirin accelerate it.

According to our data, aspirin did not alter the average wrinkle depth at the concentration of $5 \mathrm{mM}$, whereas $50 \mathrm{mM}$ of aspirin markedly increased the average wrinkle depth (Figure $3 \mathrm{C}$ ). However, skin fold thickness and epidermal thickness were decreased by the treatment of both $5 \mathrm{mM}$ and 50 $\mathrm{mM}$ of aspirin, showing that aspirin treatment caused skin thinning at both concentrations (Figures 2C and 3C). In addition, expression levels of $p 53$ and $p 16$ were also elevated by the treatment of $5 \mathrm{mM}$ of aspirin, verifying at molecular levels that $5 \mathrm{mM}$ of aspirin accelerated the skin aging (Figure $4 \mathrm{C})$. For the reason of this discrepancy, we assume that the thinning of skin precedes and wrinkle formation appears later in the course of intrinsic skin aging.

It is now uncertain how COX-2 inhibitors modulate the skin aging. Nonetheless, we have here suggested that COX-2 inhibitors modulate the skin aging by regulation of type I procollagen and caveolin-1 expression. We observed that NS-398 increased the type I procollagen expression while celecoxib and aspirin decreased it (Figure 5). Compatible with these results, it has been reported that NS-398 enhances type I procollagen expression in HDFs and promotes fibrosis in rat muscle (Han et al., 2004; Shen et al., 2005). In addition, celecoxib reduces type I collagen expression in mouse mammary gland and rat liver (Paik et al., 2009; Lyons et al., 2011). Aspirin has been reported to reduce collagen biosynthesis in HDFs (Surazynski et al., 2004). Since type I collagen is the major constituent of the extracellular matrix (ECM) of skin and the reduction of its content is associated with degenerative changes as seen in the intrinsic skin aging (Rittié and Fisher, 2002), our results suggest that COX-2 inhibitors might modulate the skin aging through regulation of type I procollagen expression. In addition, since type I collagen is the most abundant protein in the ECM of most tissues, there is a possibility that COX-2 inhibitors modulate aging not only in skin but also in other tissues. Further studies are required to elucidate the above possibility and the mechanism by which COX-2 inhibitors regulate the expression of type I procollagen.

Caveolae are flask-shaped vesicular invaginations in the plasma membrane and play central roles in receptor-mediated endocytosis. Caveolin-1 is the main component of caveolae, which has been proposed to be a key determinant for aging (Zou et al., 2011). Actually, the expression of caveolin-1 is enhanced not only in cellular senescence but also in brain, heart, kidney, lung and spleen in aged rats (Park et al., 2000). In addition, overexpression of caveolin-1 reduces cellular life span in mouse fibroblasts and siRNAs for caveolin-1 triggers reinitiation of DNA synthesis in senescent HDFs (Volonte et al., 2002; Cho et al., 2003). It appears that this pro-aging effect of caveolin-1 is attributable to its capability to interact with other aging-modulating 
molecules in caveolae. Under the treatment of hydrogen peroxide, caveolin-1 interacts with the antioxidant enzyme thioredoxin reductase 1 (TrxR1) to inhibit its enzymatic activity, which activates the p53/p21 pathway and premature senescence in NIH3T3 cells (Volonte and Galbiati, 2009). In addition, caveolin-1 interacts with the ubiquitin ligase $M d m-2$ to sequester it to the caveolar membrane, resulting in the activation of p53 and premature senescence in human and mouse fibroblasts (Bartholomew et al., 2009). We here reported that the aging-modulating effect of COX-2 inhibitors is closely associated with caveolin-1 expression levels (Figure 6), suggesting that the inhibitors might modulate the skin aging by regulation of caveolin-1 expression. Consistent with these findings, we have previously reported that among three selective COX-2 inhibitors studied, NS-398 inhibited caveolin-1 expression whereas the other inhibitors (celecoxib and nimesulide) enhanced the expression, which was closely associated with the senescence-modulating effect of the inhibitors in HDFs (Kim et al., 2008). Further studies are required to elucidate the mechanism by which COX-2 inhibitors regulate the expression of caveolin-1.

In conclusion, the results of this study suggest that the catalytic activity of COX-2 is not a causal factor for intrinsic skin aging as evidenced by acceleration of the skin aging by celecoxib and aspirin among three COX-2 inhibitors studied. However, it still remains to be elucidated whether COX-2 catalytic activity is involved in the intrinsic aging in other tissues. Therefore, it is necessary to investigate continuously the function of COX-2 and effects of COX-2 inhibitors at individual levels.

\section{Methods}

\section{Materials}

NS-398 and aspirin were purchased from Cayman Chemicals (Ann Arbor, MI). Celecoxib was a generous gift from Dr. S.V. Yim (Kyung Hee University, Korea). Drugs were diluted in a solution of ethanol and propylene glycol (7:3).

\section{Animals and drug treatment}

All procedures were approved by the Institutional Animal Care and Use Committee of the Kangwon National University (KIACUC-09-0017). Female SKH-1 hairless mice at 6 weeks of age were obtained from Orient Bio Inc. (Seongnam, Korea) and were acclimated in our facility for a week before the experiments. Five mice were allocated to each group and were treated with $100 \mu$ of drugs everyday for 12 weeks on the right and left side of their dorsal skin.

\section{Measurement of skin fold thickness}

Midline skin was manually pinched upward at the neck and base of the tail. Then, skin fold thickness was measured midway between the neck and hip by using a caliper (PEACOCK, Ozaki MFG Co., Tokyo, Japan) as described previously (Kim et al., 2005).

\section{Measurement of epidermal thickness}

Skin samples were fixed in $10 \%$ formalin and embedded in paraffin. Paraffin sections were stained with hematoxylin and eosin (H \& E), and epidermal thickness was measured by using the Labworks software (PerkinElmer Inc., Waltham, MA).

\section{Measurement of wrinkle grade}

Skin replicas were obtained by applying silicon rubbers (Cuderm Corp., Dallas, TX) onto the murine skin. The replicas were photographed and wrinkles were analyzed by the skin visiometer SV600 (Courage-Khazaka Electronic $\mathrm{GmbH}$, Köln, Germany).

\section{Western blot analysis}

Skin samples were frozen and ground in liquid nitrogen and then homogenized in RIPA buffer $(150 \mathrm{mM} \mathrm{NaCl}, 100$ $\mathrm{mM}$ Tris- $\mathrm{HCl}, 1 \%$ Tween-20, $1 \%$ sodium deoxycholate and $0.1 \%$ SDS) with $0.5 \mathrm{mM}$ EDTA, $1 \mathrm{mM} \mathrm{PMSF}, 10 \mu \mathrm{g} / \mathrm{ml}$ leupeptin, $10 \mu \mathrm{g} / \mathrm{ml}$ aprotinin and $1 \mu \mathrm{g} / \mathrm{ml}$ pepstatin. The lysates were resolved in SDS-PAGE and transferred to nitrocellulose membranes, and probed with appropriate antibodies. The immunoreactive protein complexes were detected by enhanced chemiluminescence (Amersham Bioscience, Boston, MA). Mouse monoclonal antibodies for p53 (Ab-6), p16 (sc-74401) and caveolin-1 (\#601406) were purchased from Oncogene Science (Cambridge, MA), Santa Cruz Biotechnology (Danvers, MA) and BD Biosciences (San Jose, CA), respectively. A rabbit polyclonal antibody specific for type I procollagen (BCO-A3001) was purchased from BioCol Inc. (Lake Forest, CA).

\section{Statistical analysis}

Comparison between two groups was tested by the student's $t$ test using the GraphPad Prism program (GraphPad Software). The difference between two groups was statistically significant if $P$ values were less than 0.05 .

\section{Acknowledgements}

This work was supported by the National Research Foundation of Korea (NRF) grant by the Korea government (MEST) through the Ageing and Apoptosis Research Center at Seoul National University (2008-0057303), Basic Science Research Program through the NRF funded by the Ministry of Education, Science and Technology (20100007188), and a grant from the National R\&D Program for Cancer Control, Ministry for Health and welfare, Republic of Korea (1020420). 


\section{References}

Alcorta DA, Xiong Y, Phelps D, Hannon G, Beach D, Barrett JC. Involvement of the cyclin-dependent kinase inhibitor $\mathrm{p} 16$ (INK4a) in replicative senescence of normal human fibroblasts. Proc Natl Acad Sci USA 1996;93:13742-7

Atadja P, Wong H, Garkavtsev I, Veillette C, Riabowol K. Increased activity of p53 in senescing fibroblasts. Proc Natl Acad Sci USA 1995;92:8348-52

Baek BS, Kim JW, Lee JH, Kwon HJ, Kim ND, Kang HS, Yoo MA, Yu BP, Chung HY. Age-related increase of brain cyclooxygenase activity and dietary modulation of oxidative status. J Gerontol A Biol Sci Med Sci 2001;56:B426-31

Barnett J, Chow J, Ives D, Chiou M, Mackenzie R, Osen E, Nguyen B, Tsing S, Bach C, Freire J, et al. Purification, characterization and selective inhibition of human prostaglandin $\mathrm{G} / \mathrm{H}$ synthase 1 and 2 expressed in the baculovirus system. Biochim Biophys Acta 1994;1209:130-9

Bartholomew JN, Volonte D, Galbiati F. Caveolin-1 regulates the antagonistic pleiotropic properties of cellular senescence through a novel Mdm2/p53-mediated pathway. Cancer Res 2009;69:2878-86

Braidy N, Guillemin GJ, Mansour H, Chan-Ling T, Poljak A, Grant R. Age related changes in NAD+ metabolism oxidative stress and Sirt1 activity in wistar rats. PLoS One 2011;6: e19194

Campisi J. Senescent cells, tumor suppression, and organismal aging: good citizens, bad neighbors. Cell 2005; 120:513-22

Ching TT, Chiang WC, Chen CS, Hsu AL. Celecoxib extends C. elegans lifespan via inhibition of insulin-like signaling but not cyclooxygenase-2 activity. Aging Cell 2011;10:506-19

Cho KA, Ryu SJ, Park JS, Jang IS, Ahn JS, Kim KT, Park SC. Senescent phenotype can be reversed by reduction of caveolin status. J Biol Chem 2003;278:27789-95

Cho KA, Park SC. Caveolin-1 as a prime modulator of aging: a new modality for phenotypic restoration? Mech Ageing Dev 2005;126:105-10

Chung HY, Cesari M, Anton S, Marzetti E, Giovannini S, Seo AY, Carter C, Yu BP, Leeuwenburgh C. Molecular inflammation: underpinnings of aging and age-related diseases. Ageing Res Rev 2009;8:18-30

Chung JH, Seo JY, Choi HR, Lee MK, Youn CS, Rhie G, Cho $\mathrm{KH}$, Kim KH, Park KC, Eun HC. Modulation of skin collagen metabolism in aged and photoaged human skin in vivo. J Invest Dermatol 2001;117:1218-24

Dimri GP, Lee X, Basile G, Acosta M, Scott G, Roskelley C, Medrano EE, Linskens M, Rubelj I, Pereira-Smith O, et al. A biomarker that identifies senescent human cells in culture and in aging skin in vivo. Proc Natl Acad Sci USA 1995;92: 9363-7

Donato AJ, Black AD, Jablonski KL, Gano LB, Seals DR. Aging is associated with greater nuclear NF kappa B, reduced I kappa $B$ alpha, and increased expression of proinflammatory cytokines in vascular endothelial cells of healthy humans. Aging Cell 2008;7:805-12
Dorszewska J, Adamczewska-Goncerzewicz Z. Oxidative damage to DNA, p53 gene expression and p53 protein level in the process of aging in rat brain. Respir Physiol Neurobiol 2004;139:227-36

Flower RJ. The development of COX2 inhibitors. Nat Rev Drug Discov 2003;2:179-91

Funk CD. Prostaglandins and leukotrienes: advances in eicosanoid biology. Science 2001;294:1871-5

Han JH, Roh MS, Park CH, Park KC, Cho KH, Kim KH, Eun HC, Chung JH. Selective COX-2 inhibitor, NS-398, inhibits the replicative senescence of cultured dermal fibroblasts. Mech Ageing Dev 2004;125:359-66

Herbig U, Ferreira M, Condel L, Carey D, Sedivy JM. Cellular senescence in aging primates. Science 2006;311:1257

Johnson JL, Wimsatt J, Buckel SD, Dyer RD, Maddipati KR. Purification and characterization of prostaglandin $\mathrm{H}$ synthase-2 from sheep placental cotyledons. Arch Biochem Biophys 1995;324:26-34

Kim HH, Lee MJ, Lee SR, Kim KH, Cho KH, Eun HC, Chung $\mathrm{JH}$. Augmentation of UV-induced skin wrinkling by infrared irradiation in hairless mice. Mech Ageing Dev 2005;126: 1170-7

Kim HJ, Kim KW, Yu BP, Chung HY. The effect of age on cyclooxygenase-2 gene expression: NF-kappaB activation and IkappaBalpha degradation. Free Radic Biol Med 2000; 28:683-92

Kim JW, Baek BS, Kim YK, Herlihy JT, Ikeno Y, Yu BP, Chung HY. Gene expression of cyclooxygenase in the aging heart. J Gerontol A Biol Sci Med Sci 2001;56:B350-5

Kim SR, Park JH, Lee ME, Park JS, Park SC, Han JA. Selective COX-2 inhibitors modulate cellular senescence in human dermal fibroblasts in a catalytic activity-independent manner. Mech Ageing Dev 2008;129:706-13

Krishnamurthy J, Torrice C, Ramsey MR, Kovalev GI, Al-Regaiey K, Su L, Sharpless NE. Ink4a/Arf expression is a biomarker of aging. J Clin Invest 2004;114:1299-307

Kulju KS, Lehman JM. Increased p53 protein associated with aging in human diploid fibroblasts. Exp Cell Res 1995;217: 336-45

Lyons TR, O'Brien J, Borges VF, Conklin MW, Keely PJ, Eliceiri KW, Marusyk A, Tan AC, Schedin P. Postpartum mammary gland involution drives progression of ductal carcinoma in situ through collagen and COX-2. Nat Med 2011;17:1109-15

Massie HR, Williams TR, lodice AA. Influence of antiinflammatory agents on the survival of Drosophila. J Gerontol 1985;40:257-60

Paik YH, Kim JK, Lee JI, Kang SH, Kim DY, An SH, Lee SJ, Lee DK, Han KH, Chon CY, Lee SI, Lee KS, Brenner DA. Celecoxib induces hepatic stellate cell apoptosis through inhibition of Akt activation and suppresses hepatic fibrosis in rats. Gut 2009;58:1517-27

Park EJ, Kwon TK. Rottlerin enhances IL-1 $\beta$-induced COX-2 expression through sustained p38 MAPK activation in MDA-MB-231 human breast cancer cells. Exp Mol Med 2011; 43:669-75 
Park WY, Park JS, Cho KA, Kim DI, Ko YG, Seo JS, Park SC. Up-regulation of caveolin attenuates epidermal growth factor signaling in senescent cells. J Biol Chem 2000;275:20847-52

Rittie L, Fisher GJ. UV-light-induced signal cascades and skin aging. Ageing Res Rev 2002;1:705-20

Rouzer CA, Marnett LJ. Non-redundant functions of cyclooxygenases: oxygenation of endocannabinoids. J Biol Chem 2008;283:8065-9

Sanguino E, Roglans N, Alegret M, Sanchez RM, VazquezCarrera M, Laguna JC. Prevention of age-related changes in rat cortex transcription factor activator protein- 1 by hypolipidemic drugs. Biochem Pharmacol 2004;68:1411-21

Shen W, Li Y, Tang Y, Cummins J, Huard J. NS-398, a cyclooxygenase-2-specific inhibitor, delays skeletal muscle healing by decreasing regeneration and promoting fibrosis. Am J Pathol 2005;167:1105-17

Shoulders MD, Raines RT. Collagen structure and stability. Annu Rev Biochem 2009;78:929-58

Smith WL, Garavito RM, DeWitt DL. Prostaglandin endoperoxide $\mathrm{H}$ synthases (cyclooxygenases)-1 and -2. J Biol Chem 1996;271:33157-60

Surazynski A, Palka J, Wolczynski S. Acetylsalicylic acid-dependent inhibition of collagen biosynthesis and beta1-integrin signaling in cultured fibroblasts. Med Sci

\section{Monit 2004;10:BR175-9}

Varani J, Warner RL, Gharaee-Kermani M, Phan SH, Kang S, Chung JH, Wang ZQ, Datta SC, Fisher GJ, Voorhees JJ. Vitamin A antagonizes decreased cell growth and elevated collagen-degrading matrix metalloproteinases and stimulates collagen accumulation in naturally aged human skin. J Invest Dermatol 2000;114:480-6

Volonte D, Zhang K, Lisanti MP, Galbiati F. Expression of caveolin-1 induces premature cellular senescence in primary cultures of murine fibroblasts. Mol Biol Cell 2002;13: 2502-17

Volonte D, Galbiati F. Inhibition of thioredoxin reductase 1 by caveolin 1 promotes stress-induced premature senescence. EMBO Rep 2009;10:1334-40

Wang C, Jurk D, Maddick M, Nelson G, Martin-Ruiz C, von Zglinicki T. DNA damage response and cellular senescence in tissues of aging mice. Aging Cell 2009;8:311-23

Zdanov S, Bernard D, Debacq-Chainiaux F, Martien S, Gosselin K, Vercamer C, Chelli F, Toussaint O, Abbadie C. Normal or stress-induced fibroblast senescence involves COX-2 activity. Exp Cell Res 2007;313:3046-56

Zou H, Stoppani E, Volonte D, Galbiati F. Caveolin-1, cellular senescence and age-related diseases. Mech Ageing Dev 2011;132:533-42 